\title{
SINERGIA
}

REVISTA DO INSTITUTO DE CIÊNCIAS ECONÔMICAS, ADMINISTRATIVAS E CONTÁBEIS (ICEAC)

\section{ESTUDO DA TRANSMISSÃO DE PREÇOS ENTRE O MERCADO NACIONAL E INTERNACIONAL DE ÓLEO BRUTO DE SOJA PARA O PREÇO PAGO AO PRODUTOR BRASILEIRO (1996-2016)}

\author{
ELEN PRESOTTO \\ CLAILTON ATAÍDES DE FREITAS* \\ PATRÍCIA BATISTELLA \\ LAUANA ROSSETTO LAZARETTI***
}

\begin{abstract}
RESUMO
$\mathrm{Na}$ teoria econômica desde Marshall (1985), a partir dos postulados da Lei do preço Único, acredita-se que há transmissão de preços entre mercados, parte-se do princípio que, no longo prazo, esses preços tendem a convergir para um único preço. Para tanto, este estudo tem por objetivo testar a hipótese de Assimetria de Transmissão de Preços (ATP) em função dos mercados nacional e internacional de óleo bruto de soja para o produtor rural de soja em grão brasileiro, no período de 1996 a 2016. Assim, recorre-se à econometria de séries temporais, mais especificamente, ao Modelo Vetorial de Correção de Erros para se alcançar as estimativas da ATP. Entre os principais resultados, destacam-se a cointegração entre os processos estudados, a presença de ATP para as variações do preço de óleo bruto de soja no mercado internacional e nacional, em relação ao preço pago ao produtor de soja brasileiro. Além disso, foi possível constatar, também, a existência de ATP em diferentes versões, como a espacial, vertical e de curto e longo prazo, para os mercados estudados. Os resultados encontrados podem auxiliar o produtor de soja brasileiro, principalmente na tomada de decisão em função do processo de transmissão de preços detectado. $O$ mesmo sofre influências assimétricas de preços que podem derivar da ocorrência de uso de poder de mercado.
\end{abstract}

Palavras-chave: mercado internacional e nacional; Transmissão de Preços; VECM.

\section{ABSTRACT}

In economic theory since Marshall (1985), from the postulates of the Single Price Act, it is believed that the transmission of prices between markets, assumes that prices tend to converge to a single price. The objective of this study was to test the hypothesis of exchange rate (APT) analysis in relation to the national and international soybean markets for the production of soybeans, from 1996 to 2016 . Thus, we use time series econometrics, more specifically, the Vector Error Correction Model to reach the estimates of the APT. Among the main results, we highlight the cointegration among the processes studied, the presence of APT for the variations in the price of soybean oil in the international and national markets, in relation to the price paid to the Brazilian soybean producer. In addition, it was possible to verify the existence of APT in different versions such as spatial, vertical and short and long term for the markets studied. The results found may help the Brazilian soybean producer, mainly in the decision making process due to the transmission of prices detected. It also suffers asymmetric price influences that may derive from the occurrence of market power use.

Key words: international and domestic market; Price Transmission; VECM.

Recebido em: 26-08-2018 Aceito em: 18-06-2019

\section{INTRODUÇÃO}

Nas últimas décadas, a área plantada, a produção e a produtividade da soja cresceram exponencialmente no Brasil. Entre os fatores que explicam este progresso, é possível destacar: a importância dos avanços científicos e tecnológicos, dos investimentos privados em pesquisa e desenvolvimento de tecnologia, das políticas públicas de incentivo ao setor agropecuário e, também, do aumento da demanda doméstica e internacional por proteínas e óleos de origem vegetal. Essa conjunção de fatores torna a soja uma das principais commodities negociadas no mundo; com destaque, o Brasil, o segundo maior produtor mundial de soja e o detentor da maior área plantada, ficando atrás dos EUA, segundo dados da (EMBRAPA, 2016).

\footnotetext{
"Mestra em Economia e Desenvolvimento pela Universidade Federal de Santa Maria. Doutoranda em Agronegócios pela Universidade Federal do Rio Grande do Sul. E-mail: elenpresotto@yahoo.com.br

*.. Docente do Programa de Pós-Graduação em Economia e Desenvolvimento da Universidade Federal de Santa Maria.

Mestra em Economia e Desenvolvimento pela Universidade Federal de Santa Maria. Doutoranda em Agronegócios pela Universidade Federal do Rio Grande do Sul.

Mestra em Economia e Desenvolvimento pela Universidade Federal de Santa Maria. Doutoranda em Economia pela Pontifícia Universidade Católica.
} 
A produção nacional de óleo de soja é cerca de 6869 mil-ton/ano na última década. Contudo, uma parte desta produção se destina ao mercado interno. Além disso, o óleo bruto é exportado. Apesar da queda a partir de 2007, a média de exportação anual na última década é de 1760 mil-ton/ano. Nota-se que a exportação volta a crescer a partir de 2014. Isso permite dizer que, na última década, a exportação anual de óleo bruto de soja representou, em média, $25 \%$ do montante produzido internamente (ABIOVE, 2016). O Brasil exporta óleo bruto da soja para diversos países, entre os destaques estão o Irã e a China. Além disso, com os resíduos da extração do óleo bruto, produz-se o farelo de soja, o qual, também, é exportado MDIC (2017).

$\mathrm{O}$ dinamismo do comércio internacional da soja brasileira e seus derivados está diretamente ligado à crescente sofisticação na industrialização, na difusão e no desenvolvimento de tecnologia, que aperfeiçoaram a extração de componentes da soja (óleo e insumos para a fabricação de rações), utilizados para alimentação humana. É importante ressaltar que a soja e seus derivados possuem propriedades de proteína quase pura, quando concentrados ou isolados, dando-Ihe capacidade alimentar muito versáteis. Sobretudo, as proteínas vegetais de produtos derivados da soja ganham mercados como, por exemplo, na substituição do leite e ovos na produção da indústria de pães e biscoitos, (GOODMAN; SORJ; WILKINSON, 1990).

$\mathrm{Na}$ base da cadeia do complexo soja, está o produtor rural que sofre diretamente os efeitos das flutuações dos preços da soja em grão, tanto no mercado internacional quanto nos mercados derivados, como o do óleo e do farelo de soja. Assim, o objetivo geral do estudo é testar a hipótese da existência de assimetria de transmissão de preços (ATP) entre o preço da soja pago ao produtor e os mercados internacional e nacional do óleo bruto nas últimas duas décadas. A análise de assimetria de transmissão de preço (ATP) pode auxiliar em decisões estratégias do sojicultor, entre as quais, o melhor momento de realizar a compra dos insumos, o plantio e a comercialização da soja. Entretanto, conforme é apresentado com mais detalhes na Seção 3 , não foram encontrados estudos, especificamente, sobre a abordagem proposta no presente estudo.

A base teórica da ATP é a Lei do Preço Único (LPU), a qual admite, por pressuposto, o mercado integrado e concorrencial (MARSHALL, 1985). Isso permite que os bens, em distintos mercados, tenham o mesmo preço, após a equalização, principalmente, dos custos de transportes e dos custos com impostos. No longo prazo, esta interação de mercado fará com que bens idênticos, no mesmo nível de mercado, sejam vendidos ao mesmo preço em diferentes regiões. A LPU está ligada ao processo de arbitragem, responsável pela busca do equilíbrio dos preços entre os diferentes mercados regionais, confirmando, assim, a premissa de integração de mercado, conforme explicam Krugman e Obstfeld (2007).

Neste sentido, a pesquisa tem caráter quantitativo e a econometria de série tempo constitui-se como a principal ferramenta para a abordagem proposta. As equações da ATP'S foram estimadas via modelo VECM (Vector Error Correction Model). Diante desse contexto, pode-se encerrar o problema de pesquisa com uma pergunta chave: há ATP entre os preços do óleo bruto de soja no mercado internacional e nacional para os preços pagos ao produtor de soja brasileiro?

Para responder a este questionamento, objetiva-se mensurar e analisar a ATP, tanto na sua dimensão vertical (relação entre o preço pago ao produtor de soja brasileiro e o preço do óleo bruto nacional) quanto na espacial (relação expressa entre o preço pago ao produtor de soja no Brasil e o preço da cotação internacional de óleo bruto) no período de 1996 a 2016.

O presente estudo está estruturado em seis seções. Além desta introdução (Seção 1), a Seção 2 explana o referencial teórico seguido da Seção 3 com revisão da literatura; a Seção 4 trata dos aspectos metodológicos; a Seção 5 apresenta e analisa os principais resultados alcançados com as estimativas do modelo ATP; por fim, as conclusões são apresentadas na Seção 6.

\section{REFERENCIAL TEÓRICO E FUNDAMENTOS DA ATP}

O modelo teórico utilizado no presente estudo é baseado na Lei do Preço Único (LPU). Os fundamentos teóricos da transmissão de preços entre mercados datam do século XIX, como definido por Marshall (1985). O autor descreveu a integração de preços em distintos mercados, quando, com a ausência de custos de transporte entre mercados regionais, um bem idêntico apresentará um único preço. Dessa forma, quanto mais o mercado se aproxima da perfeição, maior é a probabilidade dos preços coincidirem e refletirem em um único preço, validando, então, o preço único como "lei", reconhecido na literatura como a Lei do Preço Único (LPU).

Essa lei estabelece que bens idênticos comercializados em praças diferentes, sobre livre concorrência, com ausência de custos de transporte e barreiras oficiais ao comércio, devem ser vendidos pelo mesmo preço, quando expressos na mesma moeda. Assim, o conceito de LPU, para os autores, está relacionado à inexistência de oportunidades de arbitragem. A ausência de arbitragem, por sua vez, é a premissa de que há hipótese de mercado eficiente nesse segmento. A validade da LPU é importante para o estudo do comportamento dos mercados (FEENSTRA; TAYLOR, 2008).

Seguindo os postulados da LPU, Filho (2014) destaca que a transmissão de preços entre mercados é definida quando mudanças de preços em um mercado são transmitidas a outro mercado. Nessa situação, é 
possível afirmar que os mercados estão integrados e que as mercadorias sempre vão se movimentar do mercado com menor preço para o mercado com maior preço, ocasionando uma transferência de oferta. Como, por exemplo, do mercado brasileiro para o mercado internacional, sendo esta interação que garante a igualdade de preços, ou seja, presume-se que, sempre, vai ter um arbitrador comprando no mercado de menor preço e vendendo no de maior preço.

O modelo teórico da Lei do Preço Único (LPU), desenvolvido por Mundlak e Larson (1992), define que o preço interno corresponde ao preço do mercado internacional desde que comparado na mesma moeda. Matematicamente, tem-se

$$
P_{t}^{i}=P_{t}^{e} \cdot €_{t}^{d / f}
$$

em que: $P_{t}^{i}$ é o preço interno, $P_{t}^{e}$ é o preço externo e $€_{t}^{d / f}$ é a taxa de câmbio entre a moeda nacional e a estrangeira, neste caso, R\$/US\$. A equação (1) define a transmissão simétrica de preços. Entretanto, Meyer e Von Cramon-Taubadel (2004) referem-se à ATP como uma transmissão de bem-estar diferente da que é praticada sob condições de simetria. Na presença de um mercado com ATP, um grupo é beneficiado de uma variação nos preços de maneira desigual a outro grupo, ou seja, há uma alteração de bem-estar influenciada pelos movimentos dos preços. Os autores ainda definem uma das maiores causas de ATP, que é resultado, em grande parte, de exercício de poder de mercado, não há uma transferência de bem-estar entre os agentes envolvidos no mercado, mas uma perda líquida do mesmo.

Segundo Romain; Doyon; Frigon (2002), a assimetria de preços pode ser diferenciada na sua ocorrência entre o curto e longo prazo. A assimetria de curto prazo ocorre quando o efeito de uma variação de queda no preço pago ao produtor não é igual ao preço pago ao produtor quando ocorre uma variação positiva; no longo prazo, os efeitos podem ser os mesmos. Os autores ainda relatam que, no longo prazo, a assimetria pode ter um período de adaptação em função de uma variação de preços por um período de tempo.

Os estudos de ATP de Meyer e VonCramon - Taubadel (2004) apontam para diferentes manifestações de assimetria. O movimento dos preços pode ocorrer em função da magnitude e velocidade, podendo ser ainda positiva ou negativa e, por fim, em relação aos níveis de mercado, que pode ser de forma vertical ou espacial, quando os mercados estão distantes entre si.As Figuras 1 (a) e (b) retratam, graficamente, as versões de ATP por magnitude e por velocidade de transmissão, respectivamente.

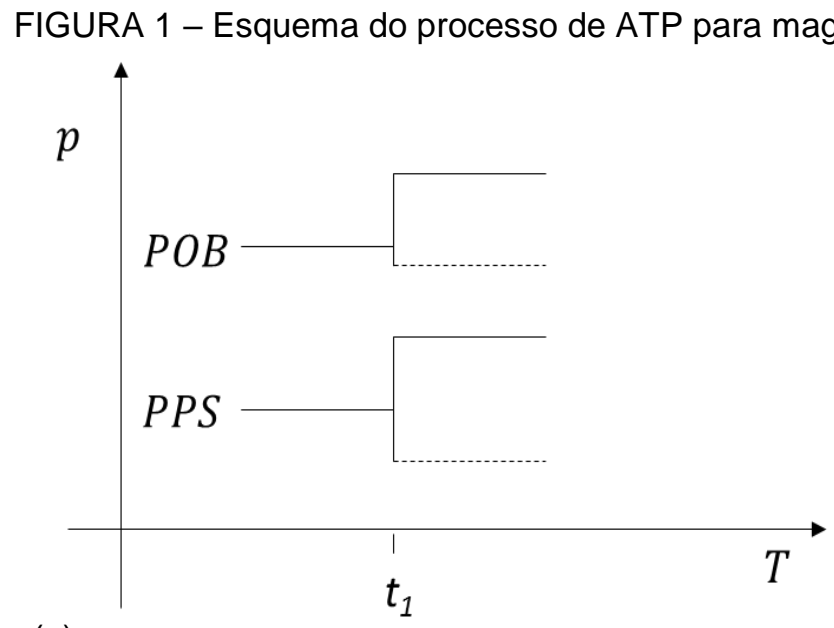

(a)

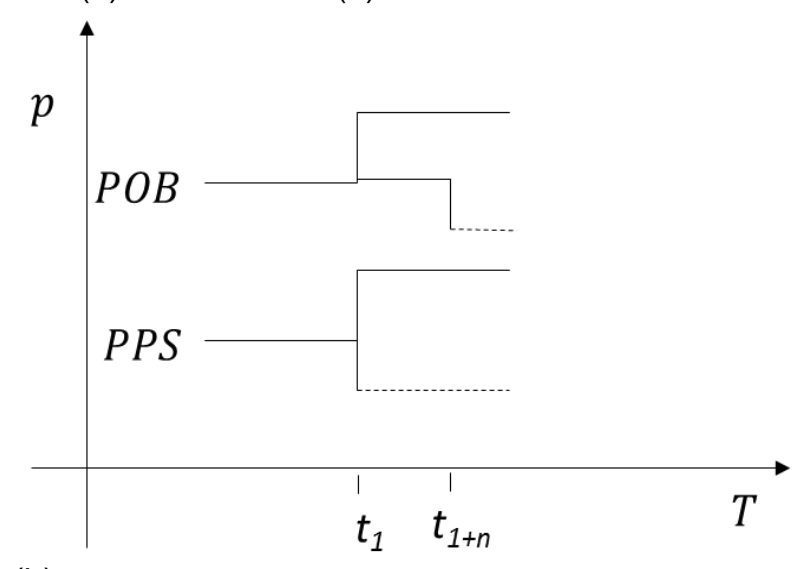

FONTE: adaptado de Meyer e Von Cramon-Taubadel (2004)

Na Figura 1 (a), a transmissão de preços para a magnitude é percebida, tendo, como exemplo, o preço de saída do preço do óleo bruto $(P O B)$, representado, no presente estudo, pelos preços do óleo bruto na CBOT e no mercado nacional; na entrada $(P P S)$, os preços pagos ao produtor de soja em grão. Nota-se que a redução do preço pago ao produtor não tem a mesma magnitude de redução em relação aos preços de saída, ou seja, a intensidade (magnitude) de redução é maior nos preços pagos ao produtor, como pode ser visualizada na Figura 1(a), pela linha pontilhada em $(P P S)$, mais baixa que em $(P O B)$.

A Figura 1 (b) representa a velocidade de transmissão de preços, ou seja, a redução do preço pago ao produtor em $t_{1}$ é repassada ao mercado de óleo bruto, por exemplo, em $t_{1+n}$, ou seja, a velocidade de transmissão, também, é diferente, representada nessa figura pela linha pontilhada, em que o preço pago ao produtor $(P P S)$ sofre a queda em $t_{1}$ e o preço de saída $(P O B)$ a queda é em $t_{1+n}$.

Sobre o segundo critério, Meyer e Von Cramon-Taubadel (2004) classificaram a transmissão assimétrica nos preços em negativa ou positiva, isto é, se o preço de saída reage mais rápido aos aumentos 
nos preços de entrada, do que para suas respectivas quedas, a assimetria é considerada como positiva, e, caso contrário, a assimetria será negativa. Em síntese, o produtor sofre influências com maior intensidade pela variável somatória negativa do que pela positiva. O terceiro critério, destacado pelos autores supracitados refere-se à transmissão assimétrica nos preços entre níveis de mercado que pode ser do tipo vertical ou espacial, entre mercados espacialmente separados. A Tabela 1 resume as manifestações de ATP e suas características que podem ser observadas para a análise de ATP.

TABELA 1 - Resumo dos tipos de assimetrias

\begin{tabular}{c|c|lc}
\hline Tipos & $\begin{array}{c}\text { Manifestação } \\
\text { de ATP }\end{array}$ & \multicolumn{1}{c}{ Descrição } \\
\hline $\begin{array}{c}\text { Intensidade } \\
\text { de Variação }\end{array}$ & $\begin{array}{l}\text { A ATP de magnitude caracteriza-se quando a redução do preço em um } \\
\text { magnitude e } \\
\text { velocidade } \\
\text { agente de mercado não tem a mesma magnitude de redução em relação } \\
\text { aos preços praticados no mercado. A velocidade de transmissão } \\
\text { assimétrica está presente no mercado quando a redução do preço em } t_{1} \text { é } \\
\text { repassada a outro mercado, por exemplo, em } t_{1+n}\end{array}$ \\
\hline $\begin{array}{c}\text { Origem da } \\
\text { variação }\end{array}$ & $\begin{array}{l}\text { positiva e } \\
\text { negativa } \\
\text { variações de preços. Se as variações de preços negativas fossem } \\
\text { repassadas com maior intensidade, caracterizar-se-ía como ATP } \\
\text { negativa; ao contrário disso,caso as variaçóes positivas fossem } \\
\text { repassadas com maior intensidade caracterizar-se-ía como ATP positiva. }\end{array}$ \\
\hline $\begin{array}{c}\text { Condição } \\
\text { de mercado }\end{array}$ & $\begin{array}{l}\text { vertical e } \\
\text { espacial } \\
\text { diferentes tipos de mercado, como produtor, atacado e varejo. A ATP } \\
\text { espacial se caracteriza quando a análise é feita em mercado } \\
\text { espacialmente separados. }\end{array}$ \\
\hline
\end{tabular}

FONTE: elaborado pelos autores.

Alguns autores como Azzam (1999) argumentam que a origem da assimetria espacial de preços ocorre na presença de um comportamento, entre os agentes, de otimização, ou seja, a assimetria é gerada e mantida no mercado em função do comportamento dos varejistas em atender aos consumidores em inúmeros mercados. Porém, no momento da decisão os consumidores, vão optar em função do custo de oportunidade e preços de ir a um lugar mais longe ou comprar mais perto da sua localização.

\section{REVISÃO DE LITERATURA}

$\mathrm{Na}$ literatura, são encontrados diversos estudos que tratam desta temática, porém, na maioria dos casos, a transmissão de preços é considerada como simétrica. E os estudos que testam a presença de assimetria em mercados, em sua grande maioria, estão ligados ao setor atacado e varejo de combustíveis. Um dos primeiros estudos internacionais com a ótica voltada para bens agropecuários é o estudo de Griffith e Piggott (1994), os quais estudaram a ATP no mercado australiano de carnes, ou seja, o preço pago ao produtor reage de forma diferente com a alta, ou com a queda do preço da carne. Diagnosticaram a assimetria de transmissão de preços, nas carnes de bovino e cordeiro, mas o mesmo não ocorreu nos preços da carne suína. A frequência dos dados foi mensal para o período de 1971 a 1988.

Tifaoui e Cramon-Taubadel (2016) constaram a existência de assimetria de transmissão de preços no mercado de manteiga na Alemanha. Usando modelo VECM, testaram a hipótese de que os preços de vendas temporárias (preços promocionais) são capazes de influenciar nos resultados da análise de transmissão vertical de preços. Usando um conjunto de dados semanais de 2005 a 2010, compararam as estimativas de transmissão vertical de preços do atacado ao nível de varejo (correspondem aos preços de venda a varejo bruto, com os preços de varejo filtrados). Os resultados confirmam que os preços de venda temporários (preços promocionais) aumentam a velocidade e a assimetria da transmissão vertical dos preços. Esses resultados agregam uma causa potencial de assimetria, como o poder de mercado. Em particular, os preços de venda temporários tornam a transmissão de preços vertical mais rápida e aumentam a probabilidade de que ela pareça assimétrica.

Em nível nacional, alguns estudos merecem destaque, como o de Aguiar e Santana (2002). Analisaram, com dados mensais de 1987 a1998, a concentração do mercado e a ATP de produtos agrícolas. Para isso, dividiram os produtos agrícolas em três grupos (perecíveis comercializados em mercados competitivos; armazenáveis negociados em mercados concentrados e armazenáveis negociados em mercados competitivos). Os autores destacaram que existem dois argumentos para explicar a assimetria de preços, a saber: a concentração do mercado e a capacidade de armazenamento do produto. Além disso, relataram que os padrões que determinam assimetria na transmissão de preços são difíceis de distinguir. Os autores consideraram não ser possível diagnosticar com precisão a hipótese de que o tipo de armazenamento e o tipo de mercado determinavam a ATP no estudo realizado. 
Neto e Parré (2012), usando dados com frequência mensal de 03/2003 a 06/2009, encontraram evidência empírica de ATP no mercado de carne bovina brasileira. O modelo utilizado para a análise foi o Structural Vector Autoregression (S-VAR). Puderam constatar a existência de ATP entre pecuaristas (produtores) e atacadistas, pois os preços comportaram-se de forma diferente diante de aumentos e quedas nos preços no elo imediatamente anterior da cadeia de carne bovina.

Andreotti (2013) estudou as regiões produtoras de raiz de mandioca e o mercado do atacado (fécula), sendo possível mensurar a influência das variações dos preços da fécula nos preços pagos aos produtores (raiz). A ATP, também, foi testada em função das regiões produtoras, ou seja, testou-se ATP vertical, procurando responder qual região tinha maior intensidade de transmissão de preço para 0 período de fev. 2002 a fev. 2013. Os resultados encontrados, obtidos através do modelo VECM, apontam para a existência de ATP e indicam relações assimétricas entre os preços da raiz e da fécula para a maioria das regiões analisadas.

Figueiredo; Souza Filho; Paullilo (2013) analisaram a presença de ATP entre os preços do suco de laranja para os preços da laranja, por meio do modelo de Mínimos Quadrados Não-Lineares, no período de jan. 2001 a mar. 2011. Os autores constataram que as empresas processadoras repassam apenas decréscimos de preços para os produtores no curto prazo e que há ATP no longo prazo.

No entanto, ainda há uma carência de estudos que avaliem o processo de transmissão de preços entre o mercado nacional e internacional de óleo de soja para o produtor de soja nacional. De modo que, até onde se conhece, ainda não foi mensurado.

\section{METODOLOGIA}

Nesta seção, é apresentado o referencial metodológico proposto para alcançar os objetivos do presente estudo e as principais considerações acerca dos conceitos da ATP. Segundo Peltzman (2000) relata em condições de ATP, há a ocorrência de rigidez na redução dos preços, ao fato que, quando o preço do óleo bruto aumenta, não necessariamente o produtor e o varejo sentem variações de mesma intensidade e direção, caracterizando, assim, a presença de ATP nos mercados estudados.

\subsection{Considerações sobre a mecânica da ATP}

Para a formalização do modelo ATP, devem ser calculadas as variáveis de aumento e de quedas dos preços. As variáveis de somatórios foram construídas a partir da diferença apresentada nos preços. Para a variável somatório positiva, caso a diferença seja positiva, então, o valor calculado é preservado, mas, quando resultar em valor negativo, a célula referente e esse valor recebe o valor zero. Considere, por exemplo, a alteração no preço do óleo bruto na CBOT, que, em janeiro de 1996, era de $\$ 540,13$, caindo em fevereiro do mesmo ano para $\$ 537,08$, ou queda de preço de $\$-3,04$. Como a diferença dos preços nos dois primeiros meses foi negativa, a célula pertinente ao aumento de preço recebe valor zero e a de queda nos preços o valor de -3,04. Assim, todas as demais informações foram calculadas. Em seguida, constrói-se a diferença positiva no mesmo sentido. Por fim, as variáveis aumento e queda representam o somatório da série diferença construída.

A partir disto, formaliza-se o VECM para a ATP na Seção 4.3, o que permite determinar em que medida as variações positivas e negativas do preço internacional e nacional do óleo bruto influenciam os preços pagos ao produtor rural de soja brasileiro.

\subsection{Especificação do Modelo de Correção de Erros (VECM)}

O VECM representa uma forma de decompor o comportamento dos componentes de curto prazo e longo prazo existentes entre variáveis da série de tempo. Além disso, é possível estimar a magnitude e rapidez de ajustamento das variáveis ao longo do tempo em função de sua trajetória de curto prazo e longo prazo. Segundo Bueno (2011), o VECM incorpora o mecanismo de correção de erro (MCE), que capta a trajetória, de longo prazo, de um processo estocástico e ajusta os desvios momentâneos de curto prazo.

A Equação (2) reflete o modelo dos impactos das variações de preços pago ao produtor de soja brasileiro em função das variações de preços da de óleo bruto nacional. $\mathrm{Na}$ especificação da parte superior dessa equação, estima-se o comportamento de curto prazo entre as variáveis. Assim, por exemplo, a variável diferenciada $\triangle P P S_{i t}$ responde às variações ocorridas nela mesma em $t-1$ e nas variações experimentadas nas demais variáveis que, também, estão diferenciadas. Os parâmetros dessas variáveis estão na matriz $\varphi$. Esse mesmo critério foi adotado para a especificação das duas outras equações de curto prazo. Na parte inferior da equação, está o modelo de longo prazo com as séries ao nível e os coeficientes 
$\alpha_{i}$ do mecanismo de correção de erros, $\beta_{i t}$ são os coeficientes da ATP de longo prazo e $\theta_{i t}$ os coeficientes da tendência do modelo. Matematicamente, são expressos por:

$$
\begin{aligned}
& {\left[\begin{array}{c}
\Delta P P S_{i t} \\
\triangle P O B A_{i t} \\
\triangle P O B Q_{i t}
\end{array}\right]=\left[\begin{array}{lll}
\varphi_{11} & \varphi_{12} & \varphi_{13} \\
\varphi_{21} & \varphi_{22} & \varphi_{23} \\
\varphi_{31} & \varphi_{32} & \varphi_{33}
\end{array}\right]\left[\begin{array}{c}
\Delta P P S_{i, t-1} \\
\Delta P O B A_{i, t-1} \\
\triangle P O B Q_{i, t-1}
\end{array}\right]}
\end{aligned}
$$

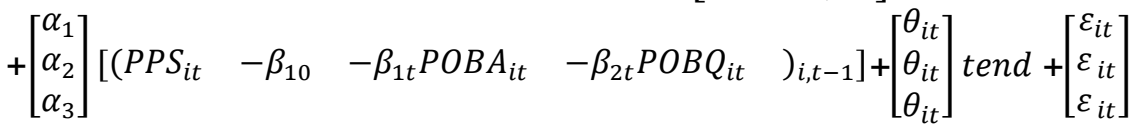

em que:

$\alpha_{i}$ : são os parâmetros estimados relativos ao mecanismo de correção de erros, ou seja, captam como a trajetória de longo prazo de um processo estocástico ajusta os desvios momentâneos de curto prazo;

$\varphi_{i t}$ : captam o comportamento autorregressivo das séries;

$P P S_{i t}$ é o preço pago pela tonelada $(1000 \mathrm{~kg})$ ao produtor rural de soja;

$P O B A_{i t}{ }^{+}$representa o somatório dos aumentos do preço pago ao óleo bruto de soja;

$P O B Q_{i t}{ }^{-}$representa o somatório das quedas do preço pago ao óleo bruto de soja;

$\theta_{i t}$ captam a tendência do modelo estimado;

tend representa a variável tendência fev. 1996=1, mar. 1996=2...;

$\varepsilon_{i t}$ representa o termo de erro do modelo proposto.

A Equação (3) reflete o modelo dos impactos das variações de preços pago ao produtor de soja brasileiro em função das variações de preços da de óleo bruto na CBOT. A interpretação dos componentes dessa equação é semelhante ao descrito na Equação (3). Matematicamente,

$$
\begin{aligned}
& {\left[\begin{array}{c}
\Delta P P S_{i t} \\
\triangle P C B O T A_{i t} \\
\triangle P C B O T Q_{i t}
\end{array}\right]=\left[\begin{array}{lll}
\Omega_{11} & \Omega_{12} & \Omega_{13} \\
\Omega_{21} & \Omega_{22} & \Omega_{23} \\
\Omega_{31} & \Omega_{32} & \Omega_{33}
\end{array}\right]\left[\begin{array}{c}
\triangle P P S_{i, t-1} \\
\triangle P C B O T A_{i, t-1} \\
\triangle P C B O T Q_{i, t-1}
\end{array}\right]+}
\end{aligned}
$$

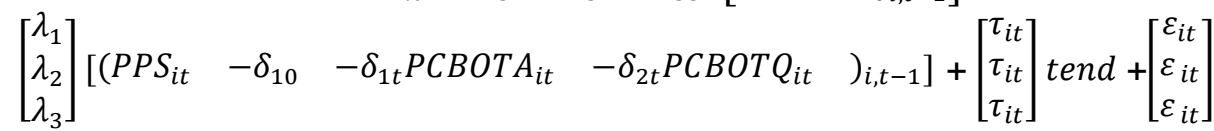

em que:

$\lambda_{i}$ : são os parâmetros estimados relativos ao mecanismo de correção de erros, ou seja, captam a trajetória, de longo prazo, de um processo estocástico e ajustam os desvios momentâneos de curto prazo;

$\Omega_{i t}$ : captam o comportamento autorregressivo das séries;

$\tau_{i t}$ catam a tendência do modelo estimado;

$P P S_{i t}$ p é o preço pago pela tonelada $(1000 \mathrm{~kg})$ ao produtor rural de soja;

PCBOT ${ }_{i t}{ }^{+}$representa o somatório dos aumentos da cotação internacional do óleo bruto da soja na CBOT; ${ }_{P C B O T Q_{i t}}{ }^{-}$representa o somatório das quedas na cotação internacional do óleo bruto da soja na CBOT.

Os outros componentes da Equação (3) seguem a definição já estabelecida na Equação (2).

\subsection{Procedimentos Econométricos}

Com a construção das variáveis conforme explicado procedimento retratado na Tabela 1, pode-se propor o modelo estruturado para a ATP. A construção das variáveis do somatório é base para validar o modelo ATP. O teste $t$ é apropriado para determinar se existem diferenças significativas na soma dos coeficientes da fase de aumentos dos preços agrícolas em relação à fase de quedas dos preços agrícolas, conforme Griffith e Piggott (1994).

Segundo Wooldridge (2010), o teste $t$ de restrição visa diagnosticar se o parâmetro do modelo é estatisticamente igual ao outro. Testa-se a hipótese $H_{0}: \widehat{\Phi_{11}}=\widehat{\Phi_{12}}$ e a hipótese alternativa, $H_{1}: \widehat{\Phi_{11}} \neq \widehat{\Phi_{12}}$, ao rejeitar-se $H_{0,}$, pode-se dizer que existe assimetria de transmissão de preços. Neste estudo, calcula-se a existência de ATP tanto no curto como no longo prazo.

Para a confirmação ou não da ATP, testes adicionais foram realizados como o de estacionariedade e o de cointegração entre os mercados do óleo bruto de soja (CBOT e doméstico) e o preço pago ao produtor de soja.

Ao se estudar a trajetória de um processo estocástico, é imprescindível verificar se existe ou não a presença de raiz unitária em tal processo. O presente estudo utilizou os testes de estacionariedade DFA (Dickey Fuller Aumentado) e NG Perron. 
O teste de DFA tem por objetivo testar a existência de raízes unitárias com vetor autorregressivo $\Delta y=\gamma y_{t-1}+u_{t}$, em que $\gamma=\rho-1$. O teste tem por hipóteses $H_{0}: \gamma=0$ e $H_{1}: \gamma<0$. Se a hipótese nula for rejeitada, então, a série temporal $y_{t}$ é estacionária; caso contrário, a série é não estacionária (tem raiz unitária).

O teste NG Perron é uma evolução dos testes de estacionariedade e apresenta melhores propriedades estatísticas vis-à-vis ao teste de DFA. Cabe destacar que, além de testar a parte autorregressiva do processo em questão, o teste NG Perron também testa a parte das médias móveis. Bueno (2011) assinala as hipóteses do teste $H_{0}: y_{t} \sim \mathrm{I}(1)$ contra a hipótese alternativa $H_{1}: y_{t} \sim \mathrm{I}(0)$. Esse teste é definido por algumas estatísticas como $\mathrm{Mz}_{\alpha}, \mathrm{Mz}_{\mathrm{t}}$ e MSB. Para a utilização da janela ótima de defasagens NG e Perron, indicam a utilização do método MAIC (Modified AIC). O teste é definido por $M z_{\mathrm{t}}: M z_{\alpha} \times M S B=z_{t}+1 / 2 \sqrt{\frac{\sum_{t=1}^{T} y_{t-1}^{2}}{v_{A R}^{2}}}(\hat{\alpha}-1)^{2}$, em que $v_{A R}^{2}$ é o estimador de longo prazo, trazendo ganhos de tamanho consideráveis, usando este estimador.

Confirmada a ordem de integração de cada processo, passa-se para o teste de cointegração. De uma forma genérica, busca-se observar que $y_{t}-\beta_{x_{t}}$ seja um processo I(0). Se afirmativo, pode-se confirmar que $y_{t}$ e $x_{t}$ são cointegrados e $\beta$ é o parâmetro de cointegração. Para analisar essa relação de cointegração, usa-se o teste de cointegração de Johansen, o qual tem, por hipótese nula, a inexistência de cointegração, e a hipótese alternativa, caso contrário. Essas hipóteses são testadas através das estatísticas do traço ou do máximo autovalor. $O$ número de raízes características $\lambda_{t r}$ diferentes de zero na matriz $\beta$, cada um dos $\lambda_{t r}$ corresponderá ao número de vetores de cointegração $(r)$. Matematicamente, a estatística do teste do traço é definida pela Equação (4).

$$
\lambda_{t r}(r)=-T \sum_{i=r+1}^{n}\left(1-\hat{\lambda}_{i}\right)
$$

em que:

$\lambda_{t r}$ é a i-ésima raiz característica, são ordenadas da maior para a menor $\left(\lambda_{1}>\lambda_{2}>\cdots \lambda_{n}\right)$; $r$ : relações de cointegração;

$\mathrm{T}=$ número de observações.

O teste tem como hipóteses formuladas por $H_{0}: r=r^{*}$ e $H_{1}: r>r^{*}$, ou seja, a hipótese nula testa se 0 número de vetores de cointegração devem ser inferiores ou igual a $r$, quanto mais distantes de zero forem os valores de $\lambda_{t r}$, mais elevado será o valor da estatística. A estatística do teste do máximo autovalor é definido pela Equação (5). Matematicamente,

$$
\lambda_{t r}(r)=-T \ln \left(1-\hat{\lambda}_{r+1}\right)
$$

O teste do máximo autovalor tem, como hipóteses formuladas $H_{0}: r=r^{*}$ e $H_{1}: r=r^{*}+1$, em que a hipótese nula testa o número de vetores de cointegração ser $r$ e a hipótese alternativa de $r+1$.

Por fim, testa-se a autocorrelação de estatística Ljung-Box.Neste caso, as hipóteses a serem consideradas são $H_{0}$ para ausência autocorrelação dos resíduos, e $H_{1}$ caso contrário, caso não se rejeite $H_{0}$, os resíduos são não correlacionados (BUENO, 2011).

\subsection{Fonte de dados}

Os dados referentes ao preço pago ao produtor de soja e a cotação internacional e nacional do óleo bruto da soja foram obtidos na ABIOVE (2016). A frequência dos dados é mensal e abrange o período compreendido entre jan. 1996 a jun. 2016 As variáveis estão mensuradas em toneladas, e os preços nacionais estão em R $\$$ /ton, e os da CBOT estão mensurados em US\$/ton.

\section{RESULTADOS E DISCUSSÕES}

A seguir, serão apresentados e discutidos os resultados obtidos a partir da estimação das Equação 2 para a análise de ATP entre o preço pago ao produtor de soja e o mercado nacional de óleo bruto e a Equação 3 para o estudo de ATP, entre o preço pago ao produtor de soja e o mercado internacional de óleo bruto, via VECM, bem como os testes realizados para a validação do modelo de ATP. 


\subsection{Análise do modelo VECM}

Com base na Tabela 2, pode-se observar que as variáveis não são estacionárias ao nível (teste DFA), pois não é possível rejeitar $H_{0}: \gamma=0$ para nenhuma delas, a um baixo nível de significância. $\mathrm{Na}$ sequência, o teste foi realizado nas variáveis na primeira diferença e, em todas, foi possível rejeitar $H_{0}: \gamma=0$, com todos os $p$-valores, aproximadamente, iguais a zero, definindo-se, assim, a ordem I(1) de integração entre todas as variáveis estudadas, ao nível de $5 \%$ de significância.

TABELA 2 - Resultado Teste DickeyFuller Aumentado (DFA)

\begin{tabular}{cccc}
\hline Teste & DFA & DFA \\
\hline Variável & Estimação & P-Valor variável em nível & P-Valor variável na 1 1 diferença $^{-}$ \\
\hline$P P S$ & 0,1779 & 0,0000 \\
$P C B O T A^{+}$ & 0,5769 & 0,0000 \\
$P C B O T Q^{-}$ & 0,6892 & 0,0000 \\
$P O B A^{+}$ & 0,1378 & 0,0000 \\
$P O B Q^{-}$ & 0,1829 & 0,0000
\end{tabular}

A seleção de defasagens baseada no critério de informação de Akaike (AIC) e todos os $p$-valores estão em função do teste com constante e tendência.

FONTE: elaborado pelos autores.

A Tabela 3 mostra os resultados do teste de NG Perron. O teste foi realizado, primeiramente, com as variáveis ao nível, observando que todas as séries são não estacionárias pela estatística $\mathrm{Mz}_{\mathrm{t}}$. Posteriormente, o teste foi realizado na primeira diferença, e como apresentado pela estatística $\mathrm{Mz}_{\mathrm{t}}$, as variáveis demostraram-se I(1). Exemplificando, a Tabela 3 apresenta o valor calculado de $P C B O T A^{+}$de 0,82 ao nível, a $5 \%$ de significância o valor crítico é de 2,91, não se rejeitando a hipótese nula para série não estacionária na estatística $\mathrm{Mz}_{\mathrm{t}}$. Repetindo-se o teste para a variável diferenciada, o valor calculado da estatística $M z_{t}$ é de $-4,92$, ao nível de $5 \%$ de significância, com valor crítico de $-2,91$, assim, rejeita-se a hipótese de série não estacionária na primeira diferença.

TABELA 3 - Resultado Teste NG Perron

\begin{tabular}{|c|c|c|c|}
\hline Variável & & Nível & 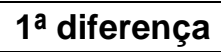 \\
\hline$P P S$ & & $M z_{t}$ & $M z_{t}$ \\
\hline Valor calculado & & -3.33949 & -5.46755 \\
\hline Valores críticos & $\begin{array}{c}1 \% \\
5 \% \\
10 \%\end{array}$ & $\begin{array}{l}-3.42000 \\
-2.91000 \\
-2.62000\end{array}$ & $\begin{array}{l}-3.42000 \\
-2.91000 \\
-2.61000\end{array}$ \\
\hline $\mathrm{PCBOTA}^{+}$ & & $\mathbf{M z _ { t }}$ & $\mathbf{M} \mathbf{z}_{\mathrm{t}}$ \\
\hline Valor calculado & & -0.82514 & -4.92598 \\
\hline Valores críticos & \begin{tabular}{r|}
$1 \%$ \\
$5 \%$ \\
$10 \%$ \\
\end{tabular} & $\begin{array}{l}-3.42000 \\
-2.91000 \\
-2.62000 \\
\end{array}$ & $\begin{array}{l}-3.42000 \\
-2.91000 \\
-2.62000 \\
\end{array}$ \\
\hline$P C B O T Q^{-}$ & & $M z_{t}$ & $M z_{t}$ \\
\hline Valor calculado & & -1.21327 & -6.35168 \\
\hline Valores críticos & \begin{tabular}{r|}
$1 \%$ \\
$5 \%$ \\
$10 \%$ \\
\end{tabular} & $\begin{array}{l}-3.42000 \\
-2.91000 \\
-2.62000\end{array}$ & $\begin{array}{l}-3.42000 \\
-2.91000 \\
-2.62000\end{array}$ \\
\hline$P O B A^{+}$ & & $\mathbf{M} \mathbf{z}_{\mathrm{t}}$ & $\mathbf{M} \mathbf{z}_{\mathrm{t}}$ \\
\hline Valor calculado & & -1.50658 & -5.24424 \\
\hline Valores críticos & $\begin{array}{r}1 \% \\
5 \% \\
10 \% \\
\end{array}$ & $\begin{array}{l}-3.42000 \\
-2.91000 \\
-2.62000 \\
\end{array}$ & $\begin{array}{l}-3.42000 \\
-2.91000 \\
-2.62000 \\
\end{array}$ \\
\hline$P O B Q^{-}$ & & $M z_{t}$ & $M z_{t}$ \\
\hline Valor calculado & & -0.94935 & -4.98257 \\
\hline Valores críticos & $\begin{array}{c}1 \% \\
5 \% \\
10 \% \\
\end{array}$ & $\begin{array}{l}-3.42000 \\
-2.91000 \\
-2.62000 \\
\end{array}$ & $\begin{array}{r}-3.42000 \\
-2.91000 \\
-2.62000 \\
\end{array}$ \\
\hline
\end{tabular}

Seleção de defasagens baseada no critério de informação de MAIC. Os resultados são apresentados para teste com constante e tendência.

FONTE: elaborado pelos autores. 
Os testes de cointegração de Johansen, pela estatística do traço e do máximo autovalor, são apresentados para o preço pago ao produtor de soja, em função das variações de preços do mercado nacional (Tabela 4) e internacional na (Tabela 5).

Os resultados da Tabela 4 também indicam um vetor de cointegração das variáveis utilizadas ao nível de $5 \%$ de significância, analisando o $p$-valor 0,00 da Tabela 4 , tanto para o teste pelo máximo autovalor como do traço, rejeita-se a hipótese de nenhum vetor de cointegração. $O$ teste para a existência de ao menos um vetor de cointegração é repetido e, com $p$-valor maior que $10 \%(0,15$ e 0,24$)$, não se rejeita a hipótese de existência de pelo menos um vetor de cointegração, segundo as estatísticas do traço e do máximo autovalor. Isso possibilita afirmar que existe uma relação estável de longo prazo, entre o preço pago ao produtor de soja e o preço no atacado nacional do óleo bruto de soja.

TABELA 4 - Resultado Teste de cointegração de Johansen/mercado interno

\begin{tabular}{cccccc}
\hline Ordem & Autovalor & Teste traço & P-Valor & Teste max.a.valor & P-Valor \\
\hline 0 & 0,42500 & 151,03 & 0,0000 & 134,47 & 0,0000 \\
1 & 0,04533 & 16,555 & 0,1521 & 11,275 & 0,2403 \\
2 & 0,02149 & 5,2800 & 0,2634 & 5,2800 & 0,2629 \\
\hline
\end{tabular}

Seleção de defasagens baseada na seleção de defasagens VAR.

FONTE: elaborado pelos autores.

Os resultados da Tabela 5 indicam a existência de cointegração das variáveis estudadas ao nível de $5 \%$ de significância, pelo baixo $p$-valor $(0,0000)$ das estatísticas do traço e do máximo autovalor. Assim, para ambos os testes, rejeita-se a hipótese de nenhum vetor de cointegração. Na sequência, testa-se existência de ao menos um vetor de cointegração, então, com p-valor maior que $10 \%(0,18$ e 0,12 , respectivamente), não se rejeita a hipótese de existência de pelo menos um vetor de cointegração. Isso possibilita estabelecer uma relação estável de longo prazo entre o preço pago ao produtor de soja e a cotação internacional do óleo bruto de soja.

TABELA 5 - Resultado Teste de cointegração de Johansen/mercado internacional

\begin{tabular}{cccccc}
\hline Ordem & Autovalor & Teste traço & P-Valor & Teste max.a.valor & P-Valor \\
\hline 0 & 0,29798 & 101,75 & 0,0000 & 85,972 & 0,0000 \\
1 & 0,05294 & 15,776 & 0,1886 & 13,219 & 0,1281 \\
2 & 0,010465 & 2,5564 & 0,6706 & 2,5564 & 0,6694 \\
\hline
\end{tabular}

Seleção de defasagens baseada na seleção de defasagens VAR.

Fonte: elaborado pelos autores.

Os dois modelos foram testados para autocorrelação pelo teste de Ljung-Box, com $p$-valor 0,53 e 0,47 , respectivamente, não se rejeitando $H_{0}$. Assim, os resíduos se comportam como ruído branco, tanto para o modelo nacional como internacional, respectivamente.

\subsection{Estimativa da ATP em função do preço do óleo bruto da soja nacional para pago ao produtor rural}

A Tabela 6 expressa resultados obtidos através da estimação do modelo VECM, para a análise de assimetria de transmissão de preços do mercado nacional de óleo bruto para o produtor de soja brasileiro.Todos os parâmetros associados ao modelo de longo prazo mostraram-se diferentes de zero ao nível de $5 \%$ de significância.É possível destacar, na segunda coluna, o resultado da influência das variáveis no longo prazo. O parâmetro da variável $P O B Q^{-}(-4,5)$ tende a influenciar negativamente o preço pago ao produtor, ou seja, quanto maior for a queda do preço do óleo nacional, menor vai ser o preço pago ao produtor de soja. Sobretudo, as quedas no preço do óleo bruto de soja nacional tendem a influenciar quedas no preço pago ao produtor de soja de $\mathrm{R} \$ 4,5$. O parâmetro da variável $P O B A^{+}(-2,35)$ indica que quanto maior for o aumento no preço do óleo bruto nacional, menor vai ser o preço pago ao produtor.Assim, os aumentos no preço do óleo bruto de soja nacional tendem a influenciar quedas no preço pago ao produtor de soja de $\mathrm{R} \$ 2,35$. 
TABELA 6 - Resultados do modelo VECM para a ATP no mercado nacional

\begin{tabular}{cccc}
\hline Variável & $\beta_{i}$ (longo prazo) & $\varphi_{i}$ (curto prazo) & $\alpha_{i}$ (vetor de erros) \\
\hline$P P S$ & 1,00 & 0,329 & $-0,003$ \\
& & $(0,074)$ & $(0,060)$ \\
$P O B A^{+}$ & $-2,357$ & 0,092 & 0,009 \\
& $(0,453)$ & $(0,049)$ & $(0,003)$ \\
$P O B Q^{-}$ & $-4,504$ & $-0,048$ & $-0,017$ \\
& $(0,542)$ & $(0,053)$ & $(0,002)$ \\
Tend & - & $-0,162$ & - \\
& & 0,097 & \\
\hline
\end{tabular}

Seleção de defasagens baseada na seleção de defasagens VAR; o modelo foi estimado sem constante.

FONTE: elaborado pelos autores.

O parâmetro $\beta_{11}$ associado à variável $P O B A^{+}$, e o $\beta_{12}$, associado à variável $P O B Q^{-}$, são responsáveis por mensurar a ATP.Cabe verificar se são ou não estatisticamente diferentes entre si, pois isso é fundamental para detectar a presença ou não de ATP. Os parâmetros da equação mostraram-se significativos para a presença de ATP no teste de $t e$ rejeitou-se a hipótese nula de $H_{0}$ : $\widehat{\beta_{11}}=\widehat{\beta_{12}}$. Sobretudo, constatou-se a existência de assimetria de transmissão de preços, pois as variações de preços negativasdo óleo tendem a ser repassadas com maior intensidade ao preço da soja do que as variações de preços positivas. Em relação ao tipo de ATP, constatou-se a existência de diferentes magnitudes, em função das quedas e aumentos de preços. As quedas nos preços do óleo se mostraram mais intensas para o preço pago ao produtor de soja. Ou seja, à medida que o preço do óleo bruto de soja nacional tem uma queda, esse valor é mais intenso para o produtor de soja, caracterizando o mercado com presença de ATP vertical e negativa.

Em relação ao curto prazo, tem-se como resultado a terceira coluna da Tabela (6). Os parâmetros representam as variáveis do estudo na primeira diferença. Com isso, pode-se ter a lógica da interação das variáveis no período anterior. Três variáveis mostraram-se significativas para explicar a variável preçoPPS $S_{i}$, sua própria variação no período anterior, ou seja, a cada mês que passa, a influência do período anterior é cerca de $\mathrm{R} \$ 0,33$ no preço pago ao produtor, ao nível de $5 \%$ de significância; a variável tendência e a variável que representa o somatório de aumento, ao nível de $10 \%$ de significância, influenciando uma queda de $\mathrm{R} \$ 0,16$ e aumento de $\mathrm{R} \$ 0,09$, respectivamente, no preço pago ao produtor de soja.

O parâmetro $\varphi_{12}$ associado à variável $P O B A^{+}$, e $\circ \varphi_{13}$ associado à variável $P O B Q^{-}$, mensuram a ATP de curto prazo. Os parâmetros da equação mostraram-se significativos para a presença de ATP. Conforme o teste de $t$ aplicado, rejeitou-se a hipótese nula, $H_{0}: \widehat{\varphi_{12}}={\widehat{\varphi_{13}}}^{2}$, logo, constatou-se a existência de ATP em que variações de preços positivas e negativas do óleo nacional tendem a ser repassadas com diferentes intensidades, ao preço pago ao produtor de soja no curto prazo.

Cabe destacar que o mecanismo de correção de erro (MCE) revelou-se significativo, estatisticamente a $10 \%$ e com sinal esperado, mas mostrou-se pouco representativo $(-0,003)$. Ou seja, a cada mês, o MCE tende a ajustar a trajetória de longo prazo em $\mathrm{R} \$ 0,003$.

\subsection{Estimativa da ATP em função do preço do óleo bruto internacional para o preço da soja pago ao produtor rural}

A Tabela 7 expressa resultados obtidos através da estimação do modelo VECM, para a análise de ATP do mercado internacional de óleo bruto para o produtor de soja brasileiro no curto e longo prazo. Todos os parâmetros do longo prazo mostraram-se diferentes de zero ao nível de $5 \%$ de significância. $O$ parâmetro $\delta_{11}$, associado à variável $P C B O T A^{+}$e o $\delta_{12}$ associado à variável $P C B O T Q^{-}$, são responsáveis por mensurar a ATP no longo prazo. É possível destacar que o parâmetro da variável $\operatorname{PCBOTQ}^{-}(-2,3)$ tende a influenciar negativamente o preço pago ao produtor, ou seja, quanto maior for a queda, menor vai ser o preço pago ao produtor de soja. Sobretudo, as quedas no preço internacional do óleo bruto tendem a influenciar uma queda do preço pago ao produtor de $\mathrm{R} \$ 2,3$. O parâmetro da variável $\operatorname{PCBOTA^{+}}(-1,09)$ indica que, quanto maior for o aumento no óleo bruto nacional, menor vai ser o preço pago ao produtor, e assim, os aumentos no preço internacional do óleo bruto tendem a influenciar uma queda do preço pago ao produtor em $\mathrm{R} \$ 1,09$.

\footnotetext{
${ }^{1}$ Teste $t: \mathrm{t}=-2,35+4,5 / 0,45-0,54=-23,88$, $\mathrm{t}$-crítico 1,96 , rejeita-se $H_{0}: \widehat{\beta_{11}}=\widehat{\beta_{12}}$ ao nível de $5 \%$ de significância

2 Teste $t: \mathrm{t}=0,092+0,048 / 0,049-0,053=-35$, t-crítico 1,96 , rejeita-se $H_{0}: \widehat{\varphi_{12}}=\widehat{\varphi_{13}}$ ao nível de $5 \%$ de significância.
} 
TABELA 7- Resultados do modelo VECM para a ATP no mercado internacional

\begin{tabular}{cccc}
\hline Variável & $\delta_{i}$ (longo prazo) & $\Omega_{i}$ (curto prazo) & $\lambda_{i}$ (vetor de erros) \\
\hline PPS & 1,00 & $-0,355$ & $-0,015$ \\
& & $(0,065)$ & $(0,065)$ \\
PCBOTA $^{+}$ & $-1,093$ & 0,163 & $-0,019$ \\
& $(0,256)$ & $(0,122)$ & $(0,004)$ \\
PCBOTQ & $-2,307$ & $-0,121$ & 0,011 \\
& $(0,365)$ & $(0,105)$ & $(0,004)$ \\
Tend & - & $-0,140$ & - \\
& & $(0,078)$ & \\
\hline
\end{tabular}

Seleção de defasagens baseada na seleção de defasagens VAR; o modelo foi estimado sem constante.

FONTE: elaborado pelos autores.

Para detectar a presença ou não de ATP no longo prazo, testa-se $H_{0}: \widehat{\delta_{11}}=\widehat{\delta_{12}}$. Os parâmetros da equação mostraram-se significativos para a presença de ATP, o teste $t$, rejeitou a hipótese nula. Sobretudo, constatou-se a existência de assimetria de transmissão de preços, pois as variações positivas têm diferentes intensidades mensuradas pelo parâmetro das variações negativas. Em relação às manifestações de ATP, constatou-se a existência de diferentes magnitudes, em função das quedas e aumentos de preços apresentarem diferentes intensidades no longo prazo. As quedas se mostraram mais influentes na transmissão de preço pago ao produtor de soja, ou seja, à medida que o preço do óleo bruto de soja no mercado internacional experimenta quedas, esta é mais intensa ao produtor de soja, caracterizando o mercado com presença de ATP espacial e negativa de longo prazo, assim como no caso do preço do óleo bruto nacional.

Em relação ao curto prazo, tem-se como resultado a segunda coluna da Tabela (7). Os parâmetros representam as variáveis do estudo na primeira diferença. Com isso, pode-se ter a lógica da interação das variáveis no período anterior. Duas variáveis mostraram-se significativas para explicar a variável preço $P P S$, no curto prazo sua própria variação no período anterior. Ou seja, a cada mês que passa, a influência do período anterior é de queda de $\mathrm{R} \$ 0,35$ no preço pago ao produtor de soja, a $5 \%$ de significância; a tendência ao nível de $10 \%$ de significância, apontando que, em média, a cada mês, o preço da soja diminui em $\mathrm{R} \$ 0,14$ no preço pago ao produtor de soja. O parâmetro $\Omega_{12}$, associado à variável $P C B O T A^{+}$e o $\Omega_{13}$ associado à variável $P C B O T Q^{-}$, mensuram a ATP de curto prazo. Os parâmetros da equação mostraram-se significativos para a presença de ATP, conforme a rejeição da hipótese nula, $H_{0}: \widetilde{\Omega_{12}}=\widehat{\Omega_{13}}$, do teste $t$. Consequentemente, as variações de preços positivas e negativas do óleo internacional tendem a ser repassadas com diferentes intensidadesrelativas ao preço para oprodutor de soja.

Cabe destacar que o mecanismo de correção de erro (MCE) se revelou estatisticamente significativo ao nível de $5 \%$ de significância, revelando-se pouco representativo $(-0,015)$, ou seja, a cada mês, o MCE tende a ajustar a trajetória de longo prazo o preço da soja em grão em $\mathrm{R} \$ 0,015$.

Em relação aos tipos de manifestações de ATP's, foram constatadas, no presente trabalho, a existência de assimetria de transmissão de preços, vertical, no caso do preço pago pela soja ao produtor em função do preço do óleo bruto nacional; espacial com relação ao preço internacional do óleo bruto e negativa para ambos os modelos no longo prazo. Assim, os parâmetros $\beta_{12}$ e $\delta_{12}$ de saída nas quedas reagem com maior intensidade aos preços de entrada (mercado nacional e internacional de óleo bruto de soja) do que os parâmetros de saídas nos aumentos de preços. De um modo mais simplificado, é o mesmo que dizer que o produtor tem uma perda de bem-estar, e, no longo prazo, não existem indícios de que a ATP desapareça para o período de tempo estudado.

No curto prazo, constatou-se a existência de ATP em ambos os casos, porém diferentemente do longo prazo, os parâmetros $\varphi_{13} \mathrm{e} \Omega_{13}$ de saída de aumentos de preços reagem com maior intensidade aos preços de entrada (mercado nacional e internacional do óleo bruto de soja) do que os parâmetros de saídas nas quedas de preços.

Esses resultados permitem entender que, em função da falta de coordenação entre os agentes envolvidos na produção, processamento e comercialização do complexo soja (Macedo, 2009), instiga os agentes a utilizarem do uso do poder de mercado (Meyer e Von Cramon-Taubadel, 2004) para obter ganhos em função das oscilações de preços de curto prazo. Nesse estudo, ainda não desapareceram, no longo prazo, resultados apontados pela análise do MCE.

Apesar de não terem sido encontrados trabalhos com propósitos convergentes aos explorados no presente estudo como o processo de transmissão entre o mercado de óleo de soja bruto nacional e internacional para o preço pago ao produtor de soja, a ATP foi detectada em todos os estudos apresentados

\footnotetext{
${ }^{3}$ Teste $t: \mathrm{t}=-1,09+2,3 / 0,25-0,36=-11$, t-crítico 1,96 , rejeita-se $H_{0}: \widehat{\delta_{11}}=\widehat{\delta_{12}}$ ao nível de $5 \%$ de significância.

${ }^{4}$ Teste $t: \mathrm{t}=0,16+0,12 / 0,12-0,10=14$, t-crítico 1,96 , rejeita-se $H_{0}: \widehat{\Omega_{12}}=\widehat{\Omega_{13}}$ ao nível de $5 \%$ de significância.
} 
na Seção 3. Para relembrá-los: Griffith e Piggott (1994), Aguiar e Santana (2002), Neto e Parré (2012), Andreotti (2013), Figueiredo; Souza Filho; Paullilo (2013), Teoh et al. (2014) e Tifaoui e Cramon-Taubadel (2016). Embora ainda seja prematuro afirmar, mas, com base na literatura encontrada, pode-se dizer que a transmissão assimétrica de preços entre mercados parece ser mais comum do que a transmissão simétrica, todavia os estudos precisam avançar neste sentido.

\section{CONCLUSÃO}

O presente trabalho se propôs a analisar o comportamento dos preços pagos ao produtor de soja em função das variações nos preços do óleo bruto de soja nos mercados doméstico e internacional. Para isso, utilizou-se o modelo VECM, estruturado nas definições de ATP (Assimetria de Transmissão de Preços), com o intuito de diagnosticar tanto a assimetria de transmissão de preços quanto os tipos de ocorrência dessa transmissão se vertical ou espacial, bem como a velocidade,a magnitude desta transmissão e 0 comportamento no curto e longo prazo.

O modelo utilizado mostrou-se eficiente para estudar a presença de ATP nos mercados analisados, em função dos testes dos parâmetros para ATP e na utilização da análise no curto e no longo prazo para mensurar a magnitude. Os resultados indicam que os mercados estudados são caracterizados por ATP do tipo vertical (transmissão assimétrica do preço do óleo bruto de soja no Brasil para o produtor de soja), espacial (transmissão assimétrica do preço do óleo bruto de soja no CBOT para o produtor de soja brasileiro) e negativa para ambos os mercados no longo prazo, já, no curto prazo, caracterizou-se ATP do tipo positiva.

O trabalho constatou que o complexo soja pode não apresentar uma coordenação entre os agentes (produtor, mercado nacional e internacional processador de soja em grão) e, de certo modo, empresas com maior participação de mercado podem empregar o poder de mercado e obter maiores ganhos na comercialização. Ao utilizarem desta vantagem, as empresas induzem a transmissão de preços assimétricos entre os agentes, as quais comercializam como produtor, varejo e consumidor final. Nestes últimos dois, já com óleo refinado.

Para futuras reflexões, sugere-se a realização de novas pesquisas ao nível de Unidades Federativas, agregando ao modelo ATP outras variáveis que afetam os preços da soja como custos de transportes, as dificuldades enfrentadas pelos produtores rurais em função de se apresentar como tomador de preços.

\section{REFERÊNCIAS}

ABIOVE. Associação Brasileira das Indústrias de Óleos Vegetais. Disponível em: <http://www.abiove.com.br/>. Acesso em: 03 jul. 2016.

ANDREOTTI, A. Q A. O poder de mercado e a formação dos preços da fécula de mandioca: uma análise para as principais regiões produtoras brasileiras. 2013. 118 p. Dissertação (Mestrado em Economia). Universidade Estadual de Maringá. Maringá, 2013.

AGUIAR, D. R.D.; SANTANA, J. A. Asymmetry in farm to retail price transmission: evidence from Brazil. Agribusiness, Vol. 18, p. 37-48, 2002.

AZZAM, A. M. Asymmetry and rigidity in farm retail price transmission. American Journal of Agricultural Economics, v. 81, p. $525-533,1999$.

BRASIL. Ministério do Desenvolvimento, Indústria e Comércio Exterior. Sistema de Análise das Informações de Comércio Exterior. Disponível em <http://www.mdic.gov.br/comercio-exterior/estatisticas-de-comercio-exterior/>. Acesso em 20.03.2017.

BUENO, R. L. S. Econometria de séries temporais. São Paulo: Cengage Learning, 2011.

FEENSTRA, R. C., TAYLOR, A. M. International Economics. Ed. Worth Publishers, $2^{2}$ ed., New York, 2008. $904 \mathrm{p}$.

FIGUEIREDO, A. M.; SOUZA FILHO, H. M.; PAULLILO, L. F. O. Análise das margens e transmissão de preços no sistema agroindustrial do suco de laranja no Brasil. Revista de Economia e Sociologia Rural, v. 51, n. 2, p. 331-350, 2013.

GOODMAN, D.; SORJ, B.; WILKINSON, J. Da lavoura às biotecnologias: agricultura e indústria no sistema internacional. Rio de Janeiro: Campus, 1990.

KRUGMAN, P. R.; OBSTFELD, M. Economia Internacional: teoria e política. 6. ed. São Paulo: Pearson Addison Wesley, 2007. $558 \mathrm{p}$

MARSHALL, A. Princípios de economia. São Paulo: Nova Cultura, 2 ed., v.2, 1985. 441 p.

MEYER, J.; CRAMON-TAUBADEL, S. Asymmetric price transmission: a survey. Journal of agricultural economics, v. 55, n. 3, p. 581-611, 2004.

NETO, W. A. S.; PARRÉ, J. L. Assimetria na Transmissão de Preços: Evidências Empíricas. Revista Econômica do Nordeste, Volume 43, № 01, Jan/Mar, 2012. 
PELTZMAN, S. Prices rise faster than they fall. Journal of political economy, v. 108, n. 3, p. 466-502, 2000.

ROMAIN, R.; DOYON, M.; FRIGON, M. Effects of state regulations on marketing margins and price transmission asymmetry: evidence from the New York city an upstate New York fluid milk markets. Agribusiness, v. 18, n. 3, p. 301-315, Spring 2002.

TIFAOUI, S.; CRAMON-TAUBADEL, S. Temporary Sales Prices and Asymmetric Price Transmission. Agribusiness, 2016. TEOH, P. S. et al. Asymmetric adjustment between crude oil and commodity market: Evidence from corn, soybeans, palm oil, wheat and sugar. 2014. Tese de Doutorado. UTAR.

WOOLDRIDGE, J. M. Introdução à Econometria. Uma Abordagem moderna. 4 ed. São Paulo: Cengage Learning, 2010. 
